\title{
ANISOTROPIES IN LUMINOSITY DISTANCE
}

\author{
NORIMASA SUGIURA, NAOSHI SUGIYAMA \\ Department of Physics, Kyoto University \\ Kyoto 606-01, Japan \\ AND \\ MISAO SASAKI \\ Department of Earth and Space Science, Osaka University \\ Toyonaka 560, Japan
}

Anisotropies in luminosity distance-redshift relation $\left(d_{L}-z\right.$ relation $)$ caused by the large-scale structure (LSS) of the universe are studied. We solve the Raychaudhuri equation on FRW models taking account of LSS by the linear perturbation method. Numerical calculations to evaluate the amplitude of the anisotropies are done on flat models with cosmological constant and open models, employing Cold Dark Matter models and COBEnormalization for the power spectrum of the density perturbations.

We found that there are three effects: peculiar velocity, gravitational lensing and Sachs-Wolfe effect. The dominant contribution is the velocity effect for low $z$ and the gravitational lensing for high $z$.

These anisotropies in $d_{L}$ cause uncertainties in determining the deceleration parameter $q_{0}$ via magnitude-redshift (equivalent to $d_{L}-z$ ) relation: $\left|\delta q_{0}\right|=\frac{2}{z}\left|\Delta d_{L} / d_{L}\right|$. The fluctuations of $d_{L}$ are amplified by the factor of $2 z^{-1}$, leading to large uncertainties of $q_{0}$ for low $z$. Our calculations give $\Delta d_{L} / d_{L} \sim 10^{-1}$ for $z=0.01,10^{-2}$ for $z=0.1$, leading to $\delta q_{0} \sim 1$ and 0.1 , respectively. This indicates that it is impossible in principle to constrain the parameter with practical presicion by observing such low $z$ samples. On the other hand, for $z=0.5$ the uncertainties of $q_{0}$ is $\sim 0.01$. Then the effect of $\Delta d_{L}$ is negligible for sources at such high redshifts. 